\title{
Real-Time Transmission and Storage of Video, Audio, and Health Data in Emergency and Home Care Situations
}

\author{
Ivano Barbieri, Paolo Lambruschini, Marco Raggio, and Riccardo Stagnaro \\ Department of Biophysical and Electronic Engineering, University of Genova, Via Opera Pia 11 A, 16146 Genova, Italy
}

Received 13 March 2006; Revised 16 January 2007; Accepted 5 March 2007

Recommended by Ying Wu

\begin{abstract}
The increase in the availability of bandwidth for wireless links, network integration, and the computational power on fixed and mobile platforms at affordable costs allows nowadays for the handling of audio and video data, their quality making them suitable for medical application. These information streams can support both continuous monitoring and emergency situations. According to this scenario, the authors have developed and implemented the mobile communication system which is described in this paper. The system is based on ITU-T H.323 multimedia terminal recommendation, suitable for real-time data/video/audio and telemedical applications. The audio and video codecs, respectively, H.264 and G723.1, were implemented and optimized in order to obtain high performance on the system target processors. Offline media streaming storage and retrieval functionalities were supported by integrating a relational database in the hospital central system. The system is based on low-cost consumer technologies such as general packet radio service (GPRS) and wireless local area network (WLAN or WiFi) for lowband data/video transmission. Implementation and testing were carried out for medical emergency and telemedicine application. In this paper, the emergency case study is described.
\end{abstract}

Copyright (c) 2007 Ivano Barbieri et al. This is an open access article distributed under the Creative Commons Attribution License, which permits unrestricted use, distribution, and reproduction in any medium, provided the original work is properly cited.

\section{INTRODUCTION}

Wired and wireless communication technologies can strongly help providing health care in several situations. Real-time transmission, storage, and retrieval of vital parameters are nowadays possible.

Electrocardiogram (ECG), blood pressure, and other patient's information can be exchanged between remote operators and specialized medical centers. Health data can be thus evaluated in real-time or offline after storage. Today, telemedicine is often applied for ill or elderly people in home care monitoring. The repetitive (daily or more frequent) monitoring of health parameters allows for the checking of long term variation and for prompt intervention. At the same time, real-time health parameter transmission in emergency situations can be crucial. In a previous project on multimedia functionalities in telemedicine systems, we used custom electronic cards and professional transmission network (e.g., Tetra). This approach is suitable (often required) for a network mainly connecting people working in the medical area. Nevertheless, in a scenario where large number of citizens ask for continuous monitoring, prompt intervention, and interaction with medical establishment, further requirements should be evaluated in order to provide new services at affordable costs. A possible solution would be to use standard equipment (less custom made as possible), commercial networks and to implement widely usable man-machine interfaces.

\section{MAIN ISSUE AND RELATED WORKS}

Telemedicine is used to be divided into three main areas: decision-making aids, remote sensing, and collaborative arrangements for real-time patients' management. Telecommunication technology allows for the supply of medical services to sites that are physically separated from the provider [1], the handling of emergencies, such as medical telemetry systems [2], and the assistance of elderly or disabled people in domestic environments. Today, available commercial systems and research prototypes emphasize how this approach to health care is rapidly evolving. A good example is the realtime remote ECG system for the monitoring of arrhythmia in a patient [3]. The system uses an event recorder and transmits to a personal digital assistant (PDA) with Bluetooth technology. Moreover, PDA tracks the patient's location via a connection to a global positioning system (GPS) receiver. 


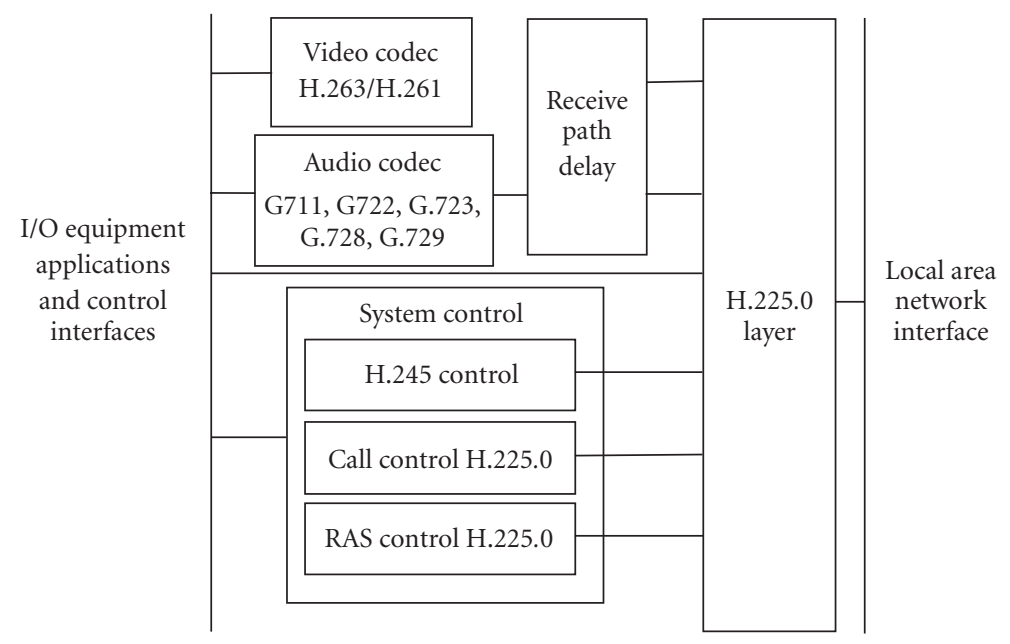

FIgURE 1: Scope of H.323 terminal equipment recommendation.

A long-distance link is established via a standard Internet connection over GSM/GPRS infrastructure. The digital signal is transmitted to a remote computer and displayed using web technology and monitored by medical professionals [3]. Another health care wireless application is the wirelessapplication-protocol-(WAP-) based telemedicine system for patient monitoring. WAP technology devices such as mobile access terminals for general inquiry and patient-monitoring services are used. Authorized users can browse patients' general data and monitor blood pressure and electrocardiogram on WAP terminals in store-and-forward mode [4]. Other application targets are emergency situations: documentation, triaging, presentation of checklists, and medical data (e.g., electrocardiograms). These critical data are transferred from the ambulance over the data network to the receiving medical facility [5]. Telemedicine also seems to be the main component of a new patient-centered health care paradigm [6] based on self-determined citizens/patients where their full involvement (as far as possible) is required during all the stages of the health care value chain, from health information and prevention all the way through to rehabilitation and long-term care. A potential advantage of including telemedicine in this environment is to give a standard and flexible response appropriately tailored both to the long and short-term health care process. Furthermore, telemedicine facilitates the continuous monitoring of health status through the transmission and storage of updated medical and general status data.

\section{SYSTEM ARCHITECTURE AND IMPLEMENTATION}

The system design follows the criteria of finding the best compromise between application requirements and available hardware and software technologies. Addressed technologies range from multimedia signal processing to human-machine interfaces (HMI). The support for health medical diagnosis, such as ECG and spirometry, is provided by interfacing the medical terminal equipment data channel to the real- time data transmission channel. From the physical connection point of view, medical devices are interfaced using both Bluetooth and RS232 serial ports. In order to allow realtime interaction and coordination among users, caregivers and medical staff, both audio and video transmission and recording require a challenging compromise between quality and bandwidth occupation. The selected video compression algorithm is H.264 [7] compliant; G.723.1 [8] is used for speech coding. The system layer follows ITU-T H.323 specification [9] (see Figure 1).

Note 1. G711, G722, and G728 are audio codec standards usable in the H.323 framework. In the described application, G.723.1 was the selected audio algorithm.

Note 2. H225 is the multiplexer standard for the H.323 specification.

Data flow through the GPRS public network which is connected to a private proprietary local area network (LAN). The Wireless Bluetooth ${ }^{\circledR}$ technology is utilized for short distance interdevice connections.

The system is composed of a set of portable units (suitcases containing a set of devices) and a Hospital central system (see Figure 3) with pc-clients and a data server interconnected with a local area network, which is linked to the external private Internet infrastructure. The portable unit communicates with the central system using a GPRS private network where the data from the portable unit are routed to a virtual private network (VPN) using a dedicated access point (APN). The portable unit system (see in Figure 4) is divided into modules which are connected as shown in Figure 2. PDA and Tablet-PC units perform data/video/audio compression, decompression, transmission, and provide HMI to the user. HMI must be the simplest possible in order to be used by health care staff [10] but also by patients who may be actively involved by their physicians in the health care and emergency delivery process [6]. 


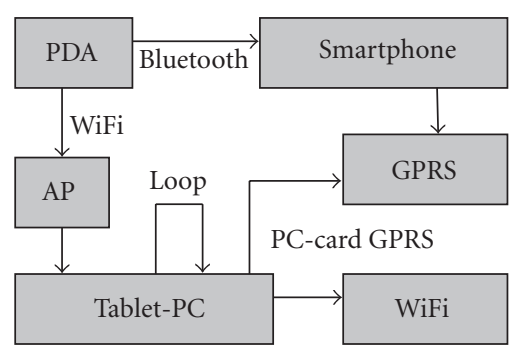

FIGURE 2: Portable unit modules interconnection.
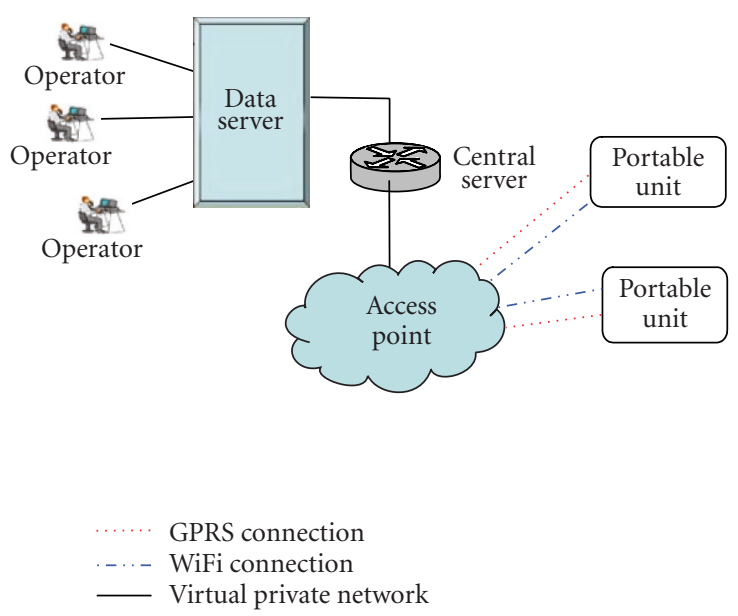

FIGURE 3: Hospital central system and hosted internal clients.

The portable unit can both transmit and store video, audio, and health parameters to the Hospital central system via GPRS link. If the GPRS connection is not available (due to environmental situation), data are stored and can be transmitted offline. In this scenario, the portable unit, with wireless access point and WiFi embedded into PDA or Tablet-PC, allows for health care operators or trained patients (or patient's relatives) to send real-time video from the place where health care processes are needed, to the suitcase that is placed in the neighborhood (usually in the caregivers medical car). The PDA acquires the video using the secure digital (SD) video camera and sends the video stream using the cellular smartphone as GPRS interface. The PDA and the smarthphone are connected through the Bluetooth interface. When the real-time live streaming is not required, data are recorded with a higher video quality. In this case, the system uses the wireless LAN instead of the GPRS public connection.

In the most common situations, the smartphone is connected to the Hospital central system using the GPRS interface.

Figure 3 shows the central system. The system can be both a hospital or a health care center. A central server receives real-time video/data. During the live media streaming any client in the center local area network can connect to the central server and play real-time audio and video. Moreover, it is also possible to search the stream database for a stored stream.

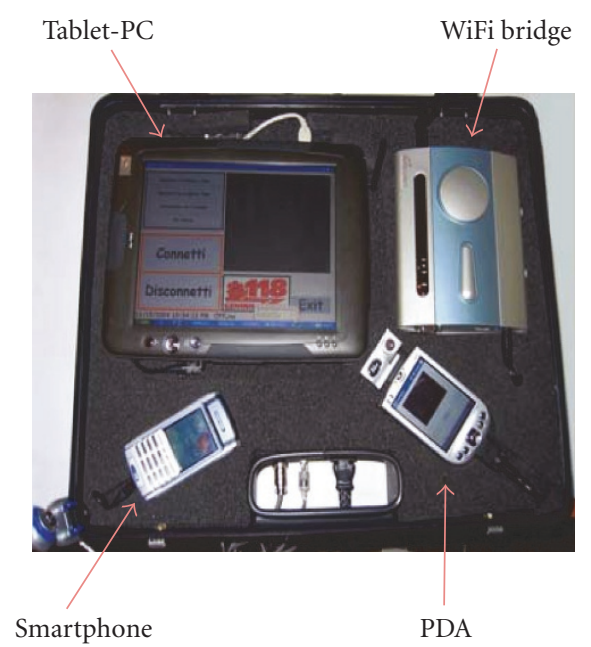

FIgure 4: Portable unit (suitcase).

The video server implements an event-driven database where each client can query for every stored diagnosis event.

HMI allows multiple real-time video stream play (up to 16 simultaneous visualizations). The central server has a separate video database administration interface. Administrators can add and remove health care multimedia streams (video, audio, or audio-video streams) events or perform other common database administrative operations. The central streaming server is also a web server allowing to query the video database through a simple web browser. The web access, protected by a user account service, is used to query the database from external-LAN users.

A multimedia real-time system design and implementation requires the best compromise between a set of critical issues related to each other with tradeoffs [11]. Windows XP Professional was chosen for the client terminals and Windows 2003 Server for the video-server application. Windows .Net technology and Visual Basic programming language were found suitable to design the HMI. For a better overall system performance a sublayer was implemented in Visual C++.

HMI functionalities should be easy to access and very intuitive in order to be used by health care staff and trained patients (elderly people at home or relatives) in critical situation. The primary target is simplicity and intuition of use. Visual Basic .Net offers facilities for the engineering of suitable interface, with useful component like info tool tips, and a very large set of available fonts for buttons and labels. Thanks to .NET flexibility, the user interface can be easily modified without affecting the underline system functionalities. This feature is used in order to adapt the access of devices to different patient typologies. The HMI main requirement is wide usability. Common users are often not familiar with PC or electronic devices. For this reason, feedback from end users was crucial. It was also important to implement an interface which could be suitable for both normal and critical/emergency situations. Figures 5 and 6 show developed client HMI. 


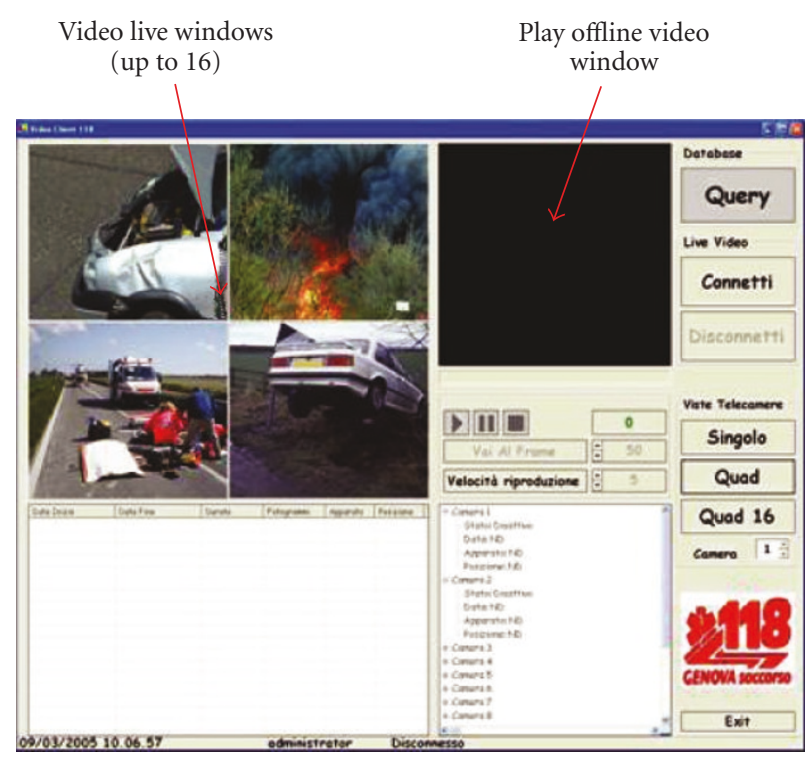

FIgURE 5: HMI for client in the hospital.

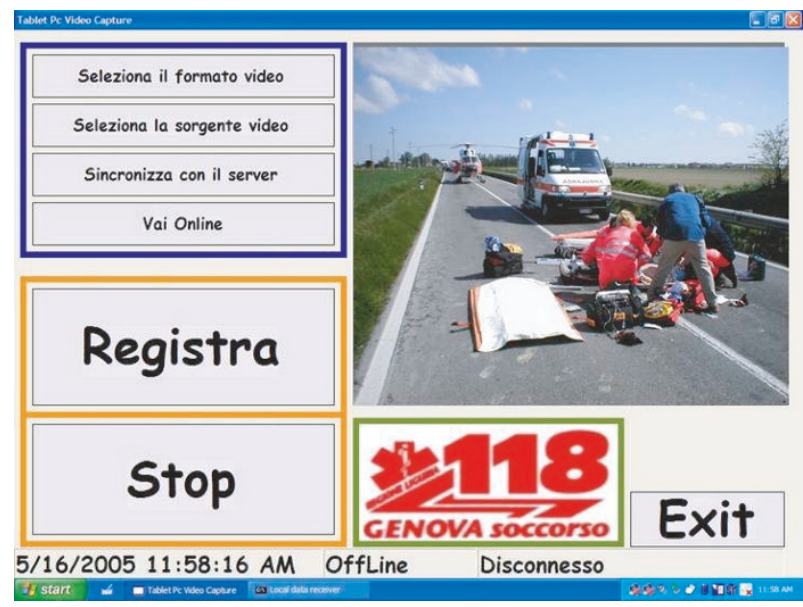

FIgURE 6: HMI of the mobile client.

The database was implemented with relational structured query Language (SQL) database. The central server can manage in real time up to sixteen data stream from portable units. This means it can contemporarily store sixteen video streams and at the same time respond to live streaming or database query client requests. A critical issue for the system was the video and audio database management. Several solutions were explored in order to find an efficient storage technique. A reliable solution was found by storing audio and video streams as separate sequences of files and not embedding them in the database. Users can retrieve multimedia streams with a transparent secure file transfer protocol (Ftp) download. Secure ftp is suitable for security and privacy of data content. This technique avoids database overload due to video/audio data relative high bit-rate. Database can therefore store long event sequences, even during tasks such as

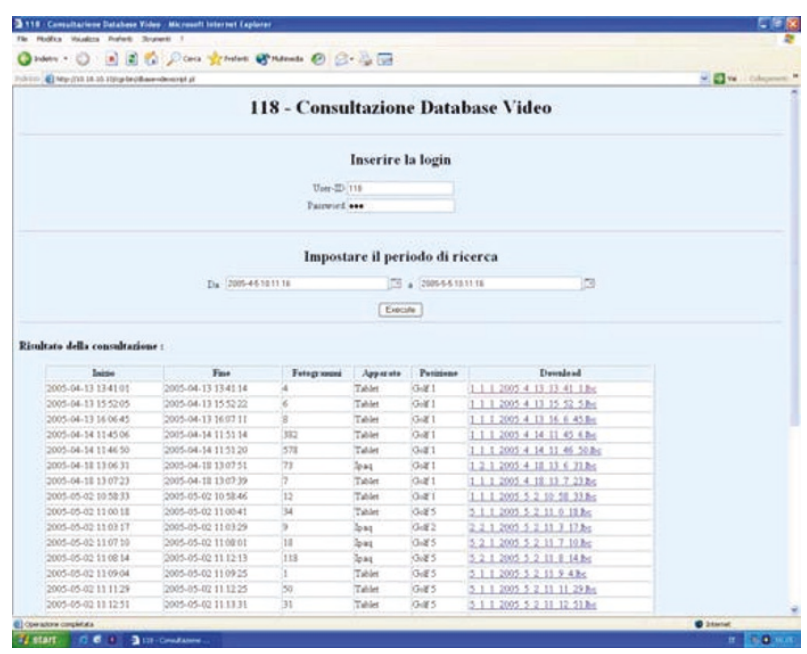

FIGURE 7: Simple web browser interface to access the multimedia database.

data reordering or data defragmentation operations. Reference data are stored in the database as small records. Figure 7 shows the web browser interface.

From the data telecommunication point of view, one of the development challenges was to reach good video/audio quality with the limited bandwidth provided by GPRS connection [12]. A long time was necessary to find the best compromise for packet length in order to achieve the required quality/bandwidth ratio. The selected and implemented codecs were ITU-T H.264 video encoder and ITUT G723.1 5.3 bps fixed bitrate speech codec. The chosen fixed rate maintains good video quality even during GPRS band lowering for bad signal coverage, with autoframe skipping during video grabbing. The G.723.1 standard algorithm also supports $6.3 \mathrm{bps}$, but the audio quality improvement together versus higher computational load did not match our application constraints. The chosen video codec performs a good video quality even when frame dropping is applied. In this situation (which is common due to GPRS band lowering), the best reachable compromise was found.

The H.264 video coding standard allows for the improving of the compression efficiency while maintaining a good video quality especially when compared with previous standards. The analysis reported in $[13,14]$ shows that the H.264 Baseline Profile achieves an average bitrate savings for video conferencing applications of about $40 \%$ compared with H.263 [15] baseline encoder. Even when compared with the H.263 apposite profile for video conferencing (conversational high compression), H.264 still saves about $28 \%$ of the bit rate. The MPEG4 [16] performance lay between the two H.263 profiles' values. Moreover, the properties of the new standard allow for the achievement of high-quality encoder effectiveness at both low and high bitrates. The ability to efficiently work at a wide range of bitrates is a crucial feature for the here-described system because of the variety of existing telemedicine applications and their future development towards the use of high-bandwidth networks. These 
characteristics, together with the network abstraction layer designed for transporting coded video data over wireless networks [17], made H.264 suitable for the designed telemedical system.

On the other hand, the computation complexity of the H.264 video coder has largely increased: as reported in [18], it has grown of more than one order of magnitude when compared with H.263 [15] and MPEG4 [16]. In order to reduce the H.264 video-encoder computational weight, a number of algorithm and software optimization were implemented. A fast motion estimation module [19] together with an adaptive interpolation module was used. Code analysis and application profiling showed these modules are the most demanding for the video codec, representing the two main performance bottlenecks. Implemented optimizations focused on the capability to adapt codec parameters to input streams. A motion detection (MD) block was integrated into the video encoder implementation in order to monitor the stream complexity and to accordingly drive the modules behavior. Specifically, MD modifies the value of the search window size parameter in the ME, whereas it changes the fractional order in the interpolation module. The optimization is based on the idea that exhaustive interpolation and motion search are useful only in video sequences containing large motions and not in low complex sequences. The obtained gain in complexity reduction is particularly favorable in slow-motion situations because of the restricted number of motion vectors we usually have in these situations. Tables 1 and 2 resume some tests where we encoded standard sequences at $30 \mathrm{fps}$ [19]. The CAVLC entropy coder and Hadamard were used for all tests, with quantization values of 28 (tests using different quantization values can be found in [19]). The H.264 activated block configurations are $8 \times 8$ and $16 \times 16$. Performance is measured in terms of time employed to compress the test sequences and the compression rate in terms of compressed sequence size. These values are compared to the reference software and the difference in percentage is shown in Tables 1 and 2. The quality is evaluated using the SNR Y, measured by the jm6.0a [20] test model (differences are shown in decibel).

From these results we observe that the proposed approach simplifies the encoder complexity achieving from $50 \%$ to $60 \%$ encoding time reduction in typical videosurveillance and video-telephony sequences. Anyway, also the worst-case foreman shows a good computational time reduction (about 25\%). It should be noted that the H.264 coding efficiency has only slightly decreased [19]. Thanks to the described modification to motion estimation we were able to exploit the remarkable bitrate versus quality baselineH.264 performance when compared to H.263 and MPEG4 $[15,16]$ with a low computational load increment. The high video quality allowed by H.264 match our application requirements where high quality video at a limited bandwidth is required.

In addition, a set of H.264 modules was implemented using optimized assembly (WMMX for the Handheld and MMX for Tablet-PC) in order to improve software performance and to decrease power consumption [21].
The audio coding implementation together with the audio/video synchronization introduces a lot of new issue such as delay, continuous play, and lip synchronization.

IP packet over the GPRS network shows variable delays and will arrive at a nonuniform rate. This effect is increased by the GPRS band lowering. Decoders must process data packets into constant streams, therefore buffers must be implemented at the receiving site [22]. We implemented a dynamic jitter buffer starting with a small size buffer increasing progressively if needed. Moreover, we decided to use an audio-priority multiplex scheme allowing to improve the perceived quality to receiver. Experimental test shows from $50 \mathrm{~ms}$ up to $200 \mathrm{~ms}$ delay for packet over the public GPRS network. We obtained further performance improvement using silence suppression to resynchronize the jitter buffer (user does not perceive audio discontinuity during silence). The average jitter buffer compensate a delay of about $100 \mathrm{~ms}$.

Information (audio, video data, and control) is sent though GPRS in several ways. Media packets (audio and video) using UDP (nonblocked) socket. Other data (controls and data such as biomedical data) are sent using TCP (blocked) socket. In order to keep audio and video synchronized the multiplex algorithm (H.225) uses time stamps.

As described above, we decided to store audio and video stream files separately for database reliability. In order to obtain synchronization, timestamps were also inserted into stored audio and video streams. If audio is present, video stream is resynchronized using timestamps for minimizing delay. For real-time transmissions, the main problem is to play a continuous audio stream, without holes or interruptions. This is accomplished with a short time prebuffering for the audio stream, which is processed at a higher priority then the video stream (audio priority).

\section{TESTING AND RESULTS}

The described system was implemented to be used in two main test applications: telemedicine in emergency handling and remote health care for patients. In this paper, the emergency case study is described. Field tests of the system were performed by regional emergency organization "(118)." Emergency staff use is definitely a meaningful test case to verify system functionalities, efficiency, and usability. From the GPRS network point of view, two different kinds of terminals were used for the test. The smartphone having $4+1$ timeslots with a CS2 coding scheme resulting in $53.6 \mathrm{kbps}$ downstream and 13.4 upstream and the pcmcia card on the TabletPC using a $4+2$ timeslots with the same downstream but an upstream of $26.8 \mathrm{kbps}$. Real-time video used QCIF frame size. The system was used for about one year, so far. Multimedia streams support was mainly employed in emergency scene description in order to better transfer urgent situation needs to the Hospital central system and to process offline (using the stored video) the protocol which is followed in emergency handling. Recorded emergency events were also used to provide the audio-video documentation of interventions. In those situations, the use of real-time multimedia streaming influences the medical protocol from a logistical 
TABLE 1: QCIF (video image format $176 \times 144$ ), quantization 28 .

\begin{tabular}{l|ccc}
\hline QCIF 28 & Time \% & Total bit \% & SNR Y $(\mathrm{db})$ \\
\hline Hall & -62.29 & 0.00 & 0.00 \\
Silent & -59.58 & +0.03 & -0.01 \\
News & -60.02 & 0.00 & 0.00 \\
Foreman & -41.57 & +0.06 & -0.02 \\
Akiyo & -62.95 & 0.00 & 0.00 \\
\hline
\end{tabular}

rather than clinical point of view. Feedback from the end user (medical staff) was collected after the test of the system. In the following lines some key points are listed. The audio stream was generally useful for emergency event recording, adding a lot of information about medical staff behavior and situation approach. About the real-time-video transmission,

(i) the video quality is acceptable even in GPRS band lowering (worst case);

(ii) the system supports decision-taking processes thanks to audio and video real-time data, thus facilitating the work of professionals located in remote hospital central system;

(iii) the real-time audio and video connection allows for a better understanding of the actual situations and feelings of patients by the remote medical staff.

While accessing offline to the audio-video-data storage,

(i) database query allows users to bypass some paper documentation, (the database storage include user identifier, ambulance id, timestamp);

(ii) hospital staff can review each moment of an emergency event to evaluate how it was handled;

(iii) the audio and video documentation allows users to give advice in legal disputations such as ambulance accidents or mistakes in patient treatment;

(iv) educational purposes: the possibility to use multimedia streams for new staff training;

(v) recorded intervention allows offline analysis to improve emergency protocol effectiveness.

For all these points, emergency staff considers the introduction of the system as a positive improvement in the handling of emergencies.

\section{CONCLUSIONS AND DEVELOPMENTS}

In this paper, a low cost system for supporting remote medical situation based on wireless connection and database storage has been described. The system makes use of real-time audio/video and medical parameters transmission and storage, in order to provide reliable and timely data to clinicians. It can be transported to handle situations where there is no radio connection coverage (GPRS). The system has been tested in actual emergency situation receiving positive feedback from the emergency staff. Further tests will focus on remote health care handling for elderly people. The use of wider bandwidth (e.g., UMTS) will improve video/audio quality and the effectiveness of data transmission. Future de-
TABLE 2: CIF (video image format $352 \times 288$ ), quantization 28 .

\begin{tabular}{l|ccc}
\hline CIF 28 & Time \% & Total bit \% & SNR Y (db) \\
\hline Hall & -67.02 & +0.03 & -0.01 \\
Silent & -60.16 & +0.03 & -0.01 \\
News & -59.52 & +0.05 & -0.02 \\
Foreman & -26.18 & +0.05 & -0.02 \\
Akiyo & -65.40 & 0.00 & 0.00
\end{tabular}

sign will address weight and size reduction in order to improve the system portability and support for a larger number of medical peripherals. Future developments will address integration of informative streams for emergency and hospitality in order for patients' history to be made quickly available.

The design criteria to implement standard protocols and algorithms following the H.323 framework outcome allow for high flexibility in system integration with last generation medical devices. Several telemedical systems are still designed according to ad hoc solutions. Usually this means that only a limited set of devices can be interfaced. Design criteria following standard recommendations will benefit not only integration issues: such standards will be included in next generation home entertainment set-top box for multimedia decoding. Thanks to the availability of higher bandwidth networking and WiFi capability for the domestic environment, the next generation set-top boxes will be the commonly available gateway interfacing domestic environment devices. In the future wireless health care sensors could therefore be interfaced in domestic environment to implement real-time health parameter acquisition in the monitoring, for example, of ill or elderly people.

\section{REFERENCES}

[1] "Telemedicine in emergency medicine," Information Paper, American College of Emergency Physicians, Dallas, Tex, USA, June 1998.

[2] E. Kyriacou, S. Pavlopoulos, D. Koutsouris, A. S. Andreou, C. Pattichis, and C. Schizas, "Multipurpose health care telemedicine system," in Proceedings of the 23rd Annual International Conference of the IEEE Engineering in Medicine and Biology Society (EMBS '01), vol. 4, pp. 3544-3547, Istanbul, Turkey, October 2001.

[3] K. J. Liszka, M. A. Mackin, M. J. Lichter, D. W. York, D. Pillai, and D. S. Rosenbaum, "Keeping a beat on the heart," IEEE Pervasive Computing, vol. 3, no. 4, pp. 42-49, 2004.

[4] K. Hung and Y.-T. Zhang, "Implementation of a WAP-based telemedicine system for patient monitoring," IEEE Transactions on Information Technology in Biomedicine, vol. 7, no. 2, pp. 101-107, 2003.

[5] R. Karlsten and B. A. Sjöqvist, "Telemedicine and decision support in emergency ambulances in Uppsala," Journal of Telemedicine and Telecare, vol. 6, no. 1, pp. 1-7, 2000.

[6] M. Pieper and K. Stroetmann, "Patients and EHRs tele home monitoring reference scenarios," in Universal Access Code of Practice in Health Telematics, C. Stephanidis, Ed., vol. 3041 of LNCS, pp. 77-87, Springer, New York, NY, USA, 2005.

[7] ISO/IEC 14496-10, ITU-T Rec.H.264, Joint Video Specification, October 2002. 
[8] ITU Rec. G.723.1: dual rate speech coder for multimedia communications transmitting at 5.3 and $6.3 \mathrm{kbit} / \mathrm{s} .03 / 96$.

[9] ITU-T Recommendation H.323-v5, "Packet based multimedia communications systems," 7/03.

[10] M. Ackerman, R. Craft, F. Ferrante, et al., "Chapter 6: telemedicine technology," Telemedicine Journal and e-Health, vol. 8, no. 1, pp. 71-78, 2002.

[11] R. B. Lee and M. D. Smith, "Media processing: a new design target," IEEE Micro, vol. 401, no. 1-2, pp. 6-9, 1996.

[12] S. N. Fabri, S. Worrall, A. Sadka, and A. Kondoz, "Real-time video communications over GPRS," in Proceedings of the 1st International Conference on $3 G$ Mobile Communication Technologie, pp. 426-430, London, UK, March 2000.

[13] J. Ostermann, J. Bormans, P. List, et al., "Video coding with H.264/AVC: tools, performance, and complexity," IEEE Circuits and Systems Magazine, vol. 4, no. 1, pp. 7-28, 2004.

[14] T. Wiegand, G. J. Sullivan, G. Bjøntegaard, and A. Luthra, "Overview of the H.264/AVC video coding standard," IEEE Transactions on Circuits and Systems for Video Technology, vol. 13, no. 7, pp. 560-576, 2003.

[15] ITU-T Recommendation H.263, "Video coding for low bitrate communication," February 1998.

[16] I. Richardson, H.264 and MPEG-4 video compression, John Wiley \& Sons, New York, NY, USA, 2003.

[17] T. Stockhammer, M. M. Hannuksela, and T. Wiegand, "H.264/AVC in wireless environments," IEEE Transactions on Circuits and Systems for Video Technology, vol. 13, no. 7, pp. 657-673, 2003.

[18] S. Saponara, C. Blanch, K. Denolf, and J. Bormans, “The JVT advanced video coding standard: complexity and performance analysis on a tool-by-tool basis," in Packet Video Workshop (PV '03), Nantes, France, April 2003.

[19] G. Bailo, M. Bariani, I. Barbieri, and M. Raggio, "Search window size decision for motion estimation algorithm in H.264 video coder," in Proceedings of International Conference on Image Processing (ICIP'04), vol. 3, pp. 1453-1456, Singapore, October 2004.

[20] JVT Reference Software version jm 6.0a, http://iphome.hhi.de/ suehring/tml/download/.

[21] N. C. Paver, "Intel wireless MMX technology," in Intel Developers Forum (IDF '02), San Jose, Calif, USA, September 2002.

[22] A. J. Hes and R. van Teeffelen, "Implementing voice over IP," The European Journal for the Informatics Professional, vol. 2, no. 3, pp. 4 pages, 2001.

Ivano Barbieri was born in Genova, Italy, in 1969. He obtained his M.S. degree in electronic engineering at Genova Universitythesis on "Research on image quality evaluation alternative methods to MSE (mean square error) in image coding systems for the subjective redundancy reduction," and Ph.D. degree - thesis on "Efficient methodologies for multimedia communication terminal design and testing." Since 1995, he

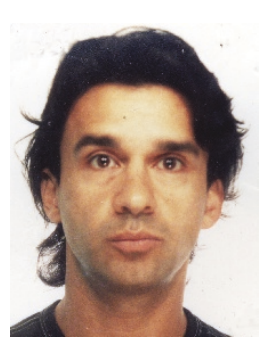
has been employed at the Department of Biophysical and Electronic Engineering (DIBE) of Genova University. Research areas are innovative approach on image quality evaluation, architectural research on systems for real-time efficient implementation of video coding algorithms exploring both embedded and single-chip solutions, real-time multimedia system (platforms, multiplexing, and control issues), DSP architecture and development environment, architecture modeling for media processing and embedded system for mobile (low power) application.

Paolo Lambruschini was born in Genova, Italy, 1974. He obtained the Laurea degree from University of Genova in 2005 writing a thesis on "Research on digital signal processor for TV signal elaboration." Presently, he is a Ph.D. student at the Department of Biophysical and Electronic Engineering at the University of Genova, Italy. Current research areas are digital signal processing for innovative power supply matrix converters, image and video coding and processing.

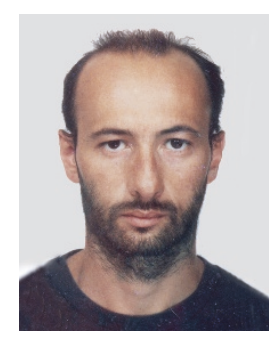

Marco Raggio was born in Chiavari (Genova), Italy, in 1964. He obtained his M.S. degree in electronic engineering at Genova University-thesis on "Development and real-time test of video compression algorithms," and Ph.D. degree-thesis on "Implementation and simulation of real-time multimedia embedded system for video telephony application and advanced DSP architecture." Since 1995, he has been em-

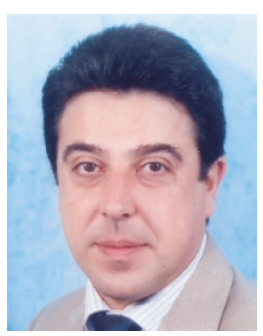
ployed as a Research Project Manager/Officer at the Department of Biophysical and Electronic Engineering (DIBE) of Genova University. In electronic system field, his interests involve hardware design and simulation, interactive real-time multimedia architecture design, that is, for mobile terminal and surveillance systems. His activities involve also field trials setup, audit, and dissemination. In networking field, he has expertise in LAN design, configuration, maintenance, security. He teaches on university seminars on video coding, standards for multimedia and streaming, DSP, industrial field bus, and embedded systems.

Riccardo Stagnaro was born in Genova, Italy, 1974. He obtained the Laurea degree from University of Genova in 2001 writing a thesis on "Study, simulation, and implementation, oriented to $\mathrm{Hw} / \mathrm{Sw}$ codesign of a motion estimation coprocessor for ITU-T H.264 video compression algorithm." Presently, he is a Ph.D. student at the Department of Biophysical and Electronic Engineering at the University of Gen-

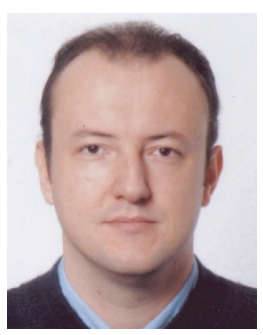
ova, Italy. Current research areas are HW/SW codesign of programmable electronic systems for real-time acquisition and processing of images and video sequences. 